\title{
Acute post-operative pain: A systematic review
}

\author{
1. Dr. SUNIL B.V. \\ Associate Professor, Department of Anaesthesiology \\ Kasturba Medical College, Mangaluru, Manipal Academy of Higher Education, \\ Karnataka, India
}

2. Dr KAVYA P

Assistant Professor, Department of Anaesthesiology

Kasturba Medical College, Mangaluru. Manipal Academy of Higher Education, Karnataka, India

3. Dr TRISHNA $P$

Assistent Professor, Department of Anaesthesiology

Kasturba Medical College, Mangaluru. Manipal Academy of Higher Education, Karnataka, India

4. Dr MAHESH NAYAK

Assistent Professor, Department of Anaesthesiology

Kasturba Medical College, Mangaluru. Manipal Academy of Higher Education, Karnataka, India

Corresponding address

Dr Sunil B V

Additional professor, anaesthesia

Kasturba medical college, mangalore, manipal academy of higher education.

$\frac{\text { drsunilbv@gmail.com }}{9620178559}$

\footnotetext{
Abstract:

Post-operative pain management is very important part of surgical care. Improper management of perioperative pain can result in increased hospital stay, cast of treatment, morbidity or mortality. This review aims to present key considerations for postoperative pain management.

Searches of the Cochrane, PubMed and Google Scholar databases were performed using terms postoperative pain, pain management, acute pain, and analgesia.

Result and conclusion. Effective treatment of acute postoperative pain is possible when we utilize a true multimodal approach which includes non-pharmacological and pharmaceutical strategies.
} 


\section{Introduction:}

Surgical procedures induces nociceptor sensitization which varies in quality, location, and intensity of postoperative pain ${ }^{1}$. Mediators released locally and systemically during and after surgery that contribute to nociceptor sensitization include: prostaglandins, interleukins, cytokines and neurotrophins. Decreased tissue $\mathrm{pH}$ and oxygen tension, and increased lactate concentration, persist at the surgical site for several days ${ }^{2}$. In neurophysiological experiments, activation and sensitization of peripheral nociceptors and spinal dorsal horn neurons are identified.

Assessment of pain can be a simple and straightforward task when dealing with acute pain and pain as a symptom. visual analogue scale (VAS) and numeric rating scale (NRS) for assessment of pain intensity agree well and are equally sensitive in assessing acute pain after surgery, along with a four point verbal categorical rating scale(VCR) ${ }^{3}$. The verbal categories mild, moderate, and severe pain may correspond to different values on the VAS in the same patient on different occasions, whereas the NRS and VAS values generally agreed well. Thus, a categorical pain scale should be used only as a coarse screening instrument, and more accurate pain intensity assessment should rely on an NRS or VAS, even in routine clinical assessment. ${ }^{4}$

Fig 1

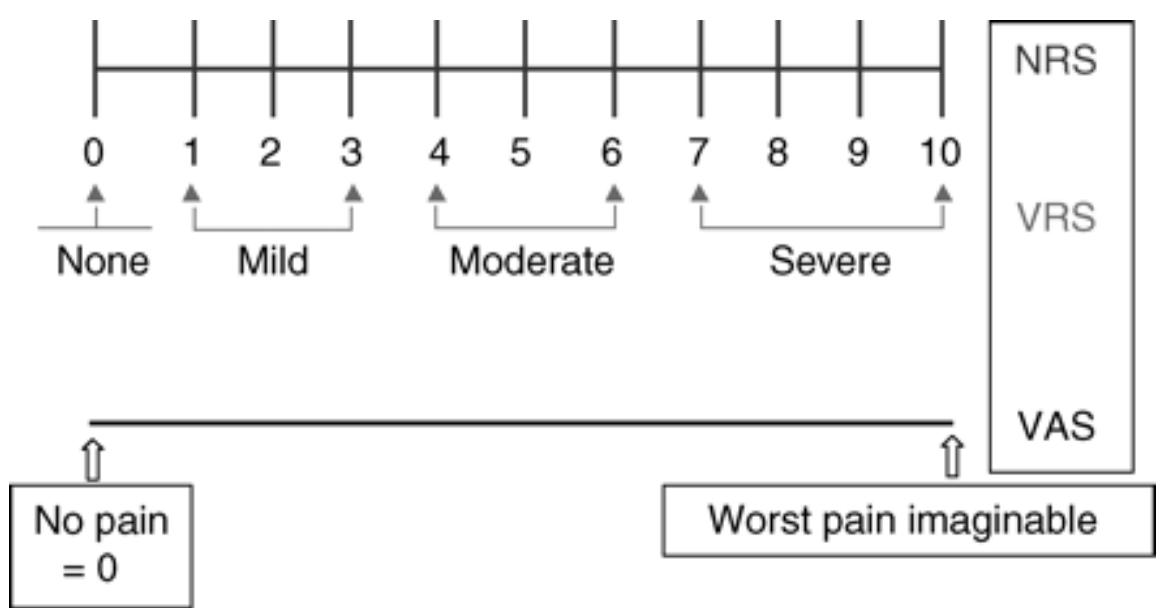

Assessment of the intensity of acute pain at rest after surgery is important for making the patient comfortable in bed. However, adequate relief of dynamic pain during mobilization, deep breathing, and coughing is more important for reducing risks of cardiopulmonary and thromboembolic complications after surgery. Effective relief of dynamic pain facilitates mobilization and therefore may improve long-term outcome after surgery. ${ }^{5}$

Nonpharmacological interventions to alleviate pain rely on the inhibition of pain signalling. ${ }^{6}$ Nonpharmacological approaches to the relief of pain are more commonly associated with nonacute settings and may be classified as follows:(i)psychological interventions (including distraction, stress management, hypnosis, and other cognitive-behavioural interventions),(ii)acupuncture and 
acupressure,(iii)transcutaneous electrical nerve stimulation,(iv)physical therapies (including massage, heat/cold, physiotherapy, osteopathy, and chiropractic).

List of pharmacological treatments utilized for the treatment of pain in postsurgical patients:

1. Pre emptive analgesia

2. Systemic pharmacologic therapy

3. Regional and Neuroxial anesthetic techniques

4. Local, Intra-articular, or topical techniques

Recent understandings in pre-emptive analgesia have defined it as an intervention given before incision or surgery, given that it is more effective than the same treatment administered after incision or surgery. ${ }^{7}$ Several pre-emptive analgesic regimens have been tried in humans. These include intravenous doses of opioids, peripheral nerve blocks, local infiltration of the surgical site, epidural administration of opioids and local anesthetics, and multimodal combinations.

Treatment with nonsteroidal anti-inflammatory drugs given systemically and NMDA receptor antagonists has also been tried pre-emptively ${ }^{8}$.

The NSAIDs comprise a heterogenous group of six major chemical classes of drugs that are primarily used as anti-inflammatory agents. The main mechanism of action of NSAIDs is inhibition of the formation of prostaglandins (as well as prostacyclin and thromboxane) from arachidonic acid via inhibition of cyclooxygenase enzymes 1 and 2 (COX-1 and COX-2, also known as prostaglandin synthase $)^{9}$. NSAIDs exert their effect through the inhibition of COX enzymes, thereby reducing the production of prostaglandins and diminishing nociceptive signal transduction. Paracetamol is one of the world's most widely used analgesics, and despite its ubiquitous use, the mechanism by which it elicits pain relief is not fully understood. The analgesic effects of paracetamol appear to be mediated through both peripheral and central mechanisms. Peripherally, the reduction in prostaglandin synthesis reduces transduction of the sensory nerves, leading to decreased nociceptive impulse transmission. Centrally, paracetamol inhibits the increase in central nervous system prostaglandins that are induced by peripheral nociceptive transmission. ${ }^{10}$

The term opioid refers to medicines derived from the opium poppy and other sources (so called synthetic or semi-synthetic), which act as opioid receptor agonists (full or partial action). The opioid class includes familiar medicines such as morphine, fentanyl, oxycodone, remifentanil, alfentanil and pethidine. Opioids are administered to control moderate to severe pain. They act by binding presynaptic opioid receptors, which prevents release of substance $\mathrm{P}$ via membrane hyperpolarization, thereby preventing impulse propagation. The majority of opioid receptors are located in the central nervous system including the spinal cord; some are found peripherally. ${ }^{11}$ In the postoperative period, opioids are administered commonly through intravenous patient controlled analgesia, or PCA.

opioid analgesia is well established and effective, but there are significant disadvantages which includes sedation and respiratory depression. Termination of nociception at the level of the spinal cord is one such technique. This may be achieved in either the subarachnoid or epidural spaces. Though continuous spinal catheters have a role in some obstetrical and 
surgical cases, the use of epidural analgesia is far more common in many countries. ${ }^{12}$ Continuous epidural infusion of local anesthetic and opioid provides superior analgesia when compared to most other techniques. Since opioid receptors are found in the substantia gelatinosa in the posterior spinal cord and these areas are anesthetized by an epidural infusion, far smaller anesthetic doses are required than when administered systemically thereby limiting unwanted effects. ${ }^{13}$ Most institutions offer a continuous infusion pump with the added function of a patient controlled bolus, which can limit the basal infusion rate.

One modality for the intervention of acute pain occurs in the periphery, at the level of the nociceptor. Local anesthetics can be used to prevent the depolarization of the nerve by blocking the sodium channel, thus preventing the pain signal's propagation from the periphery into the CNS. These medications have the benefit of completely eliminating or greatly reducing the amount of acute pain experienced. Past and current surgical practice included blocking initial pain signals by infiltration with local anesthetics or topical application of LA over mucosal or serosal membrane. Infiltrated local anesthetic has both anti-inflammatory effect as well as ability to block nerve transmission. ${ }^{14}$

Nine predictors of poor postoperative pain control were identified. They include younger age, female sex, anxiety or depression disorder, smoker, sleep disorder, pre operative pain and those on pre operative analgesia. These factors can predispose to poor control of postoperative pain. ${ }^{15}$

Newer drugs like Tapentadol, a new centrally acting analgesic that relies on a dual mechanism of action. These are mu opioid receptor agonism and norepinephrine (noradrenaline) reuptake inhibition. It shows comparable efficacy in moderate to severe pain and reduced gastrointestinal adverse events compared with fentanyl, hydromorphone, morphine, oxymorphone and oxycodone ${ }^{16}$. Co adjuvents likeAlpha-2-delta modulators (gabapentin and pregabalin) are gaining imapartence in the management of acute pain.. Perioperative pregabalin administration reduced opioid consumption and opioid-related adverse effects after surgery. ${ }^{17}$

\section{Conclusion.}

A combination of modalities should be utilized to reduce pain perception and it should be focused to each individual person. The patient with improved acute pain management yields increased satisfaction, reduced hospital stay/ costs, and decreased morbidity.

\section{References}

1. Michael A.E. Ramsay. Acute postoperative pain management. Proc (Bayl Univ Med Cent) 2000 Jul; 13(3): 244-247. doi: 10.1080/08998280.2000.1192768

2. Dahl JB, Kehlet H. Postoperative pain and its management. In: McMahon SB, Koltzenburg M, editors. Wall and Melzack's textbook of pain. Elsevier Churchill Livingstone; 2006. p 635-51.3 
3. H. Breivik, P. C. Borchgrevink, S. M. Allen, L. A. Rosseland, L. Romundstad, E. K. Breivik Hals, G. Kvarstein, A. Stubhaug, Assessment of pain, BJA: British Journal of Anaesthesia, Volume 101, Issue 1, July 2008, Pages 17-24, https://doi.org/10.1093/bja/aen103

4. Breivik EK, Björnsson GA, Skovlund E. A comparison of pain rating scales by sampling from clinical trial data, Clin J Pain, 2000, vol. 16(pg. 22-8).

5. Stubhaug A, Breivik H. Breivik H, Shipley M. Prevention and treatment of hyperalgesia and persistent pain after surgery, Pain Best Practice and Research Compendium, 2007LondonElsevier(pg. 281-8)

6. Pak S C, Micalos P S, Maria S J, Lord B .Nonpharmacological Interventions for Pain Management in Paramedicine and the Emergency Setting: A Review of the Literature. Volume 2015 .Article ID873039.https://doi.org/10.1155/2015/873039.

7. Møiniche S, Kehlet H, Dahl JB. A qualitative and quantitative systematic review of preemptive analgesia for postoperative pain relief: the role of timing of analgesia. Anesthesiology. 2002;96:725-741.

8. .Vadivelu N, Mitra S, Schermer E, Kodumudi V, Kaye A D, Urman R D. Preventive analgesia for postoperative pain control: a broader concept..Local Reg Anesth. 2014; 7: 1722.

9. Vane JR. Inhibition of prostaglandin synthesis as a mechanism of action for aspirin-like drugs. Nat New Biol [Internet]. 23 Jun 1971 [Accessed on 18 Dec 2018];231(25):232-5.

10. Graham GG, Davies MJ, Day RO, Mohamudally A, Scott KF. The modern pharmacology of paracetamol: therapeutic actions, mechanism of action, metabolism, toxicity and recent pharmacological findings. Inflammopharmacology [Internet]. 30 Jun 2013 [Accessed on 28 Dec 2018];21(3):201-32.

11. Cohen MJ, Schecter WP. Perioperative pain control: A strategy for management. Surg Clin N Am. 2005:1243-1257

12. Palmer CM. Continuous spinal anesthesia and analgesia in obstetrics. Anesthesia and Analgesia. 2010;111(6):1476-9.

13. Johnson Q, Robert R. Borsheski, Joseph L. Reeves-Viets. A Review of Management of Acute Pain. Mo Med. 2013 Jan-Feb; 110(1): 74-79.

14. Radwan IA, Shigeru S, Fumio G. The Neurotoxicity of local anesthetics on growing neurons: a comparative study of lidocaine, bupivacaine, mepivaciane, and ropivacaine. Anesthesia Analagesia. 2002;94:319-24.

15. Yong MMH, Heartly RL, Leung AA, Ronksley PE, Jetty N et al. Preoperative predictors of poor acute postoperative pain control: a systematic review and meta-analysis. BMJ open 2019 april 1;(9)4e025091. doi: 10.1136/bmjopen-2018-025091.

16. Stephan A. Schug, Catherine Goddard. Recent advances in the pharmacological management of acute and chronic pain. Annals of palliative medicine. Vol 3, No 4 (October 2014)

17. J Zhang 1, K-Y Ho, Y Wang. Efficacy of pregabalin in acute postoperative pain: a metaanalysis. Br J Anaesth. 2011 Apr;106(4):454-62. doi: 10.1093/bja/aer027. Epub 2011 Feb 26. 